\title{
Efficient Beacon Placement for Network Tomography
}

\author{
Ritesh Kumar and Jasleen Kaur \\ Department of Computer Science \\ University of North Carolina at Chapel Hill \\ \{ritesh, jasleen\}@cs.unc.edu
}

\begin{abstract}
Recent interest in using tomography for network monitoring has raised the fundamental issue of whether it is possible to use only a small number of probing nodes (beacons) for monitoring all edges of a network in the presence of dynamic routing. Past work has shown that minimizing the number of beacons is NP-hard, and has provided approximate solutions that may be fairly suboptimal. In this paper, we use a two-pronged approach to compute an efficient beacon set: (i) we formulate the need for, and design algorithms for, computing the set of edges that can be monitored by a beacon under all possible routing states; and (ii) we minimize the number of beacons used to monitor all network edges. We show that the latter problem is NP-complete and use an approximate placement algorithm that yields beacon sets of sizes within $1+\ln (|E|)$ of the optimal solution, where $\mathrm{E}$ is the set of edges to be monitored. Beacon set computations for several Rocketfuel ISP topologies indicate that our algorithm may reduce the number of beacons yielded by past solutions by more than $50 \%$.
\end{abstract}

\section{Categories and Subject Descriptors}

C.2.3 [Computer Systems Organization]: Computer-Communication Networks - Network Operations: network monitoring

\section{General Terms}

Algorithms, Management

\section{Keywords}

Network Monitoring, Tomography, Beacon Placement, Optimality

\section{INTRODUCTION}

The last two decades have witnessed an exponential growth of the Internet in terms of its infrastructure, its traffic load, as well as its commercial usage. Today, the growth of the world's economy depends heavily on the connectivity, reliability, and quality of service provided by Internet Service Providers (ISPs). The ability to monitor the health of their networks is essential for ISPs to provide

Permission to make digital or hard copies of all or part of this work for personal or classroom use is granted without fee provided that copies are not made or distributed for profit or commercial advantage and that copies bear this notice and the full citation on the first page. To copy otherwise, to republish, to post on servers or to redistribute to lists, requires prior specific permission and/or a fee.

IMC'04, October 25-27, 2004, Taormina, Sicily, Italy.

Copyright 2004 ACM 1-58113-821-0/04/0010 ...\$5.00. good service to customers. Consequently, there is significant interest in developing network monitoring infrastructures that allow ISPs to monitor their network links.

A key consideration in the design of monitoring infrastructures is to develop low-cost solutions. In particular, the idea of placing and operating sophisticated monitors at all nodes in a network is not cost-efficient. Instead, there has been significant recent interest in relying on tomographic techniques that use only a few probing nodes (beacons) for monitoring the health of all network links [1, $2,3,4,5,6,7]$. A key challenge is to find a small set of beacons that is guaranteed to be able to monitor all network links, even with dynamically-changing IP routes. Two recent efforts have focused on the problem of finding the smallest beacon sets for a network [4, 7]. These, however, do not adequately meet the above challengethe beacon set of [4] is not robust to changes in IP routes, and the beacon set proposed in [7] can be quite large for real ISP topologies (Sections 2 and 5). In this paper, we present beacon placement strategies that meet both aspects of the above challenge.

Our approach relies on a two-pronged methodology. First, we define the concept of a deterministically monitorable edge set (DMES) of a beacon as the set of edges that can be monitored by the beacon under all possible route configurations. We present efficient graph-theoretic algorithms for computing the DMES of all candidate beacons for a given network. Second, we consider the problem of finding the minimum number of beacons such that the union of their DMES covers all network edges. We show that this is an NPcomplete problem. We then use an approximate solution that yields beacon sets of sizes within $1+\ln (|E|)$ of the optimal solution, where $E$ is the set of network edges. Finally, we prove and exploit additional properties of beacons that help in improving the computational efficiency of our algorithm. Our experimental results with several real ISP topologies obtained from the Rocketfuel project [8] illustrate that our beacon placement strategy yields beacon sets that are $50-70 \%$ smaller than those yielded by [7].

The rest of this paper is organized as follows. In Section 2, we formulate the problem of beacon placement and discuss past work. In Section 3, we define and compute DMES. Section 4 discusses beacon set minimization. Section 5 presents experimental results with Rocketfuel topologies. We conclude in Section 6.

Notations and Assumptions. We model a network as an undirected graph $G(V, E)$, where $V$ is the set of network nodes and $E$ is the set of links (or edges) - in [9], we extend our analysis to directed graphs as well. We use the terms network and graph interchangeably. We assume that $G$ is connected (there exists a path from any node to any other node) and that all routes are simple (acyclic). Finally, we say that two physical paths between a pair of nodes are distinct, if they differ in even one of the edges traversed. 


\section{PROBLEM FORMULATION}

In a tomographic network monitoring infrastructure, each network link is monitored by a special probing node, referred to as a beacon. ${ }^{1}$ The basic idea behind most tomographic setups is fairly simple: the beacon sends a pair of nearly-simultaneous probes to the two end-nodes of the link, only one of which traverses the link. Each end-point sends back a response to the beacon-this may be implemented using ICMP echo messages. The results of the probes can then be used to infer properties of the link. For instance, if the objective is to measure link delays, then the difference in round-trip times of the two probes can be used as an estimate. If the objective is to simply detect link transmission failures, the success and failure of the two probes may be used as reasonable estimators.

Note that, in general, a beacon is capable of monitoring several network links. A set of beacons that can be collectively used to monitor all the links of a network is referred to as a beacon set. A central issue in the design of a monitoring infrastructure is that of beacon placement - which network nodes should be used to construct a beacon set? Two requirements guide the design of a good beacon placement strategy:

\section{- Minimizing the number of beacons.}

One of the prime motivations for using tomography for network monitoring is to reduce the cost of the monitoring infrastructure. However, even a tomographic infrastructure involves the development, installation, debugging, operation, and maintenance of specialized software/hardware on each beacon. In order to minimize the cost of doing so, it is important that the number of beacons used to monitor all links of a given network are minimized.

- Robustness to routing dynamics.

Routing state in many networks responds to changes in traffic patterns and link loads, as well as to link failures. Since Internet traffic conditions are highly dynamic, the default IP routes in a given network may change at relatively small time-scales. A monitoring infrastructure, therefore, should not assume a specific routing configuration in order to assign a beacon to a given link. More generally, a beacon set should be able to monitor all network links, independent of the current route configuration.

In this paper, we focus on the problem of beacon placement that meets the above requirements. Specifically, our objective is to: minimize the number of beacons required to deterministically monitor all the links of a given network, even in the presence of dynamism in IP routes.

\subsection{Past Work}

A fundamental step in finding the smallest beacon set for a network is to first enlist the edges that can be monitored by each candidate beacon, referred to as the monitorable edge set (MES) of the beacon. Note that the union of MES of all beacons in a beacon set is equal to the set of all network edges. In general, the larger is the average MES size in a beacon set, the smaller is the beacon set. ${ }^{2}$

\footnotetext{
${ }^{1}$ Some applications of tomography may require multiple beacons to monitor a given link [10].

${ }^{2}$ Perhaps the largest MES (and smallest beacon set) that can be envisioned is when a single beacon monitors all the links of a network - this is feasible, for instance, in a network which supports source-routing [11]. In such a network, a beacon can precisely specify the path traversed by its probes, and hence can probe the end-points of any network link. However, this strategy relies on the availability of source-routing support at all network nodes, which is the not the case with a majority of current networks [4].
}

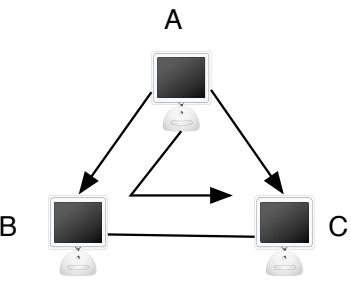

Beacon A is "locally flexible"

$\mathrm{MES}=\{\mathrm{AB}, \mathrm{AC}, \mathrm{BC}\}$

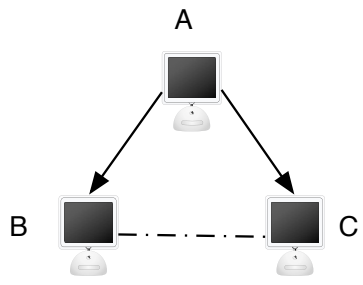

Beacon A is "simple"

$M E S=\{A B, A C\}$

\section{Figure 1: Simple vs. Locally-flexible Beacons}

Below, we briefly discuss two beacon placement schemes that have been proposed in recent literature, which differ in their assumptions about which links comprise the MES of a beacon.

- Simple Beacons: In [4], the authors assume that the MES of a beacon consists of all links that can be reached by the beacon-which are links that lie on its IP routing tree. ${ }^{3}$ In order to monitor a link in its MES, the beacon-henceforth referred to as a "simple" beacon-sends probes to the endpoints of the link, along the default IP paths to those endpoints. The authors demonstrate that the problem of minimizing the size of the beacon set with such beacons, is NPhard, and provide a placement strategy that produces a beacon set no larger than $1+\log |E|$ times the optimal beacon set. Unfortunately, since the authors assume that all links within the routing tree of a beacon belong to its MES, their strategy is not robust to changes in routing trees and works only for networks with static routes.

- Locally-flexible Beacons: In [7], the authors consider beacons that have a greater flexibility in selecting the paths taken by the probes. Specifically, the beacons-henceforth referred to as "locally-flexible" beacons-are capable of selecting the first link (outgoing link from beacon) on which a probe to any destination is transmitted. A probe can, therefore, be sent to a destination either along the current IP route to the destination, or along one of the current IP route from any immediate neighbor to the same destination (Figure 1). ${ }^{4}$ Furthermore, the authors do not assume static routing state and define the MES of a beacon to consist of links that, irrespective of what current routes are, can always be monitored. The authors do not provide a mechanism to compute such an MES for a beacon, but show that even if these sets are known, the beacon set minimization problem is NP-hard. The authors instead suggest an alternative beacon-placement strategy which, unfortunately, could result in fairly large beacon sets for current network topologies (see Section 5).

To summarize, existing beacon placement strategies ${ }^{5}$ are either not robust to routing dynamics or are inefficient in minimizing the

\footnotetext{
${ }^{3}$ The IP routing tree of a node refers to the tree, rooted at the node, which is formed by the links that lie on the default IP routes from that node to each of the other nodes in the network.

${ }^{4}$ The authors in [7] implicitly assume that the default IP route from any neighbor to the said destination will not go through the beacon node. This assumption may get violated when a path through the beacon has a smaller cost that any other physical path between a neighbor and the destination.

${ }^{5} \mathrm{An}$ orthogonal problem of beacon placement for detecting $\mathrm{mul}$ -
} 
number of beacons. In this paper, we build on past work to address these limitations by using a two-pronged approach:

1. Deterministic MES Computation: In order to be insensitive to routing dynamics, for each candidate beacon, we determine the set of edges - referred to as its Deterministic MES (DMES) - that can be monitored by it under all possible routing configurations.

2. Beacon Set Minimization: In order to minimize cost, we address the problem of finding the smallest beacon set.

In the following two sections we present our abstractions and methodologies for achieving these two steps for both simple and locally-flexible beacons.

\section{DETERMINISTICALLY MONITORABLE EDGE SETS}

The first key problem we need to solve is to find the set of edges that can be monitored by a beacon, independent of the routing configuration. This is formally captured in the following definition.

Definition 1. An edge is said to be deterministically monitorable by a beacon if the beacon can monitor it under all possible route configurations. The Deterministically Monitorable Edge Set (DMES) of a beacon is the set of all deterministically monitorable edges associated with that beacon.

In what follows, we consider both simple and locally-flexible beacons and present algorithms for computing their DMES. Below, we consider both simple and locally-flexible beacons and present methodologies for computing their DMES. We assume throughout that a beacon of either type is able to monitor all edges directly connected to it, irrespective of the routing configuration. Thus, all edges incident on a node $u$ belong to its DMES. Lemma 1, proved in [9], further establishes a crucial property of a deterministically monitorable edge (DME)

Lemma 1. If a beacon $u$ has the ability to reach, under all possible route configurations, one of the end-points of an edge $e$ through that edge, then $u$ can deterministically monitor $e$.

\subsection{DMES for Simple Beacons}

Theorem 1. Let $u$ be a simple beacon and let $S(v)$ be the set of all distinct physical paths from $u$ to another node $v$. The link $l(v)$ is deterministically monitorable by $u$ if for all paths $p$ in $S(v)$, $l(v)$ is the last edge on $p$. The DMES of $u$ is the set of all such edges $l(v)$ for all nodes $v \in V$.

Proof: Since all paths from the beacon $u$ to $v$ have $l(v)$ as the last edge, the current IP route from $u$ to $v$ (which takes one of these paths) ends in the edge $l(v)$. From Lemma 1, therefore, beacon $u$ is able to monitor the link $l(v)$.

tiple link failures that occur simultaneously has been considered in [12]. In general, it is not possible to detect all cases of simultaneous link failures in a given network. In [12], the authors restrict their attention to those simultaneous link failures that can be detected in the absence of any limitations on the number of beacons and probes. They then provide efficient algorithms for minimizing the number of beacons and probes needed for detecting these failures. Like [4], this work assumes "simple" beacons and uses the IP routing tree in the beacon set computation and, hence, is applicable only to networks with non-dynamic routes. As part of future work, we hope to use our formulations from this paper to extend the work in [12] to other beacon types and to networks with dynamic routing.

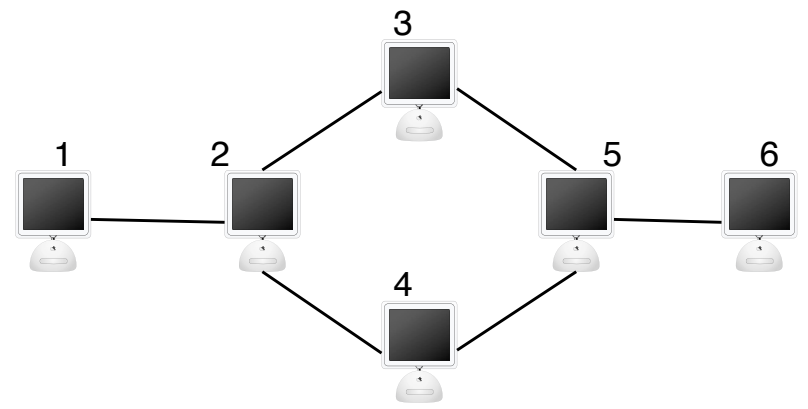

Figure 2: The DMES may not a connected graph.

Note that a DMES yielded by Theorem 1 has no more structure than an arbitrary edge set. In particular, the DMES need not form a connected sub-graph; Figure 2 illustrates that the DMES of node 1 includes the edges 1-2 and 5-6. We now present an efficient algorithm for computing the DMES for simple beacons.

Algorithm 1. Computing DMES of a simple beacon $u$.

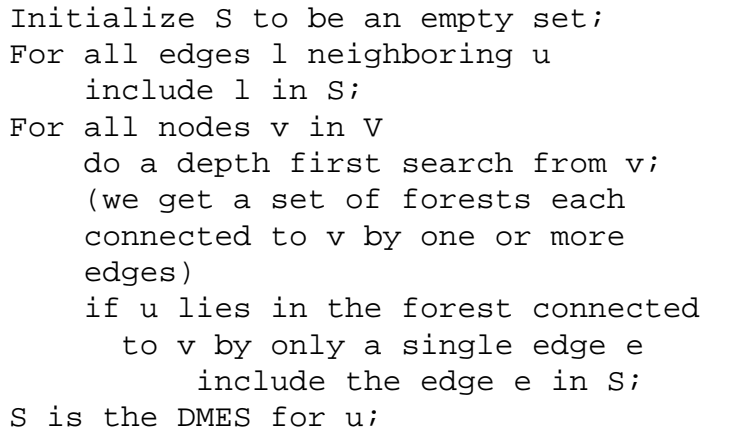

Proof: (Proof of correctness) Consider a depth first tree (along with its back edges) constructed from the node $v$. If we consider all forests rooted at the immediate neighbors of $v$, then these might connect to $v$ via one or more edges. Separating forests this way helps us to isolate all possible paths from the beacon $u$ to the node $v$. Any probe packet from $u$ to $v$ is entirely confined to paths in the forest containing $u$. Now, if the beacon $u$ lies in a forest which connects to $v$ via only one edge, all paths from $u$ to $v$ have to cross this edge at the end of the path. However, if $u$ lies in a forest which is connected to $v$ via two or more edges, then there exist at least two distinct paths from the beacon $u$ to the node $v$ which end in different edges to the node $v$. This means that the edges are not deterministically monitorable from $u$ (Theorem 1).

Time Complexity: The cost of computing the DMES of a simple beacon is essentially that of running a depth first search (DFS) algorithm at every node in the network. Since the time complexity of running a depth first search on $G(V, E)$ is $\theta(|E|+|V|)$, the time complexity of Algorithm 1 is $\theta(|V|(|E|+|V|))$.

Note that the DMES for multiple beacons can be computed in parallel. After running DFS on a node $v$, we can add an incident edges of $v$ to the DMES of all beacons that belong to the forest rooted at the edge, if there are no more edges connecting that forest to $v$. Since the number of potential beacons is bounded by $|V|$, and the time complexity of depth first search is $\theta(|E|+|V|)$, the time complexity for the parallel DMES computation algorithm is the same as above. Hence, we can calculate the DMES of all nodes in $G(V, E)$ in $\theta(|V|(|E|+|V|))$ time. 


\subsection{DMES for Locally-flexible Beacons}

Theorem 2. Let $u$ be a locally-flexible beacon and $E_{u}$ be the set of edges directly connected to $u$. For each edge $i \in E_{u}$, let $S_{i}(v)$ be the set of all paths from $u$ to any other node $v$, that start with the edge $i$. A link $l_{i}(v)$ is deterministically monitorable from $u$ if for all paths in $S_{i}(v), l_{i}(v)$ is the last edge. The DMES of $u$ is the set of all deterministically-monitorable edges $l_{i}(v)$, for all $v \in V$ and all $i \in E_{u}$.

Proof: Since locally-flexible beacons can select the outgoing link on which to transmit a probe, we need to consider only those paths to $v$ which start from a specific edge in $E_{u}$, to see if there is a common ending edge. Thus, even if $u$ has paths to $v$ which end with different edges, if all paths to $v$ that start from $u$ with edge $i$ end with a common edge $l_{i}(v), u$ has the control over the ability to reach $v$ through $l_{i}(v)$. From Definition 1 and Lemma 1, therefore, the common edge is deterministically monitorable.

Below, we present an algorithm for computing the DMES for locallyflexible beacons.

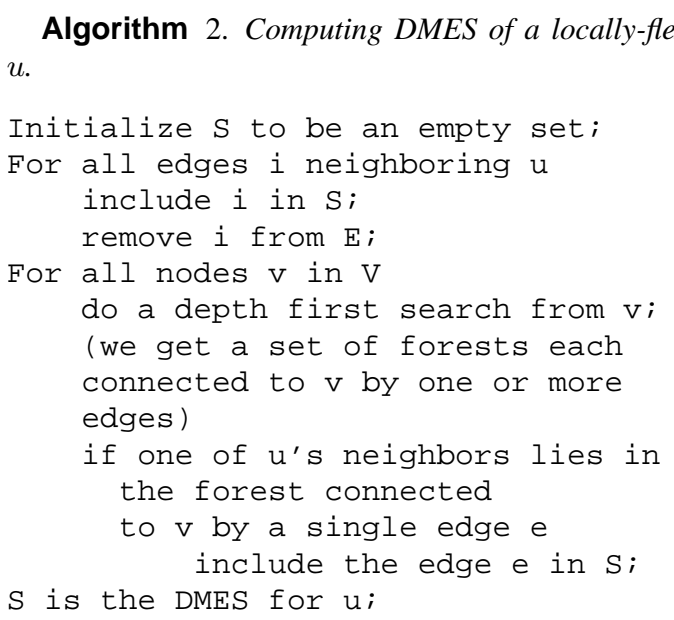

Proof: (Correctness) The proof is similar to that for Algorithm 1. Let $u_{i}$ be the neighbor connected to $u$ through $i$. The forest containing $u_{i}$ also contains all paths from $u$ to $v$ that start in $i$. This is because, if there was another path from $u$ to $v$ through $i, v$ and $u_{i}$ would have been connected via a path which would be captured in the depth first search. Conversely, consider any simple path from $u_{i}$ to $v$. Since Algorithm 2 removes $i$ from $E$, adding $i$ at the start of the path still retains the "simple" property of the path. Such a path is a valid path from $u$ to $v$ starting with edge $i$. Since we removed $u$ 's neighboring edges from $E$, one could argue that some paths might be missing. However, this cannot be true because no simple paths from $v$ to $u$ would transit $u$ in the middle of the path. Hence the forests obtained by removing the edges neighboring $u$ are representative of all paths from $u$ 's neighbors to $v$.

Time Complexity: The cost of this algorithm is that of running a depth first search on each node and for each depth first search run checking if any of the neighbors of $\mathrm{u}$ are in a singly connected forest. Thus, if the degree of $u$ is $k$, the time complexity of the algorithm is $\theta(|V|(|E|+|V|+k))$. Since $k$ is bounded by $|V|$, the time complexity is $\theta(|V|(|E|+|V|))$. Note that, unlike simple beacons, DMES can not be computed in parallel for multiple nodes because for each beacon we customize the graph $G(V, E)$ (removal of neighboring edges) specific to the beacon before doing all the depth first searches. The complexity of computing DMES for all nodes in $G(V, E)$ is, therefore, $\theta\left(|V|^{2}(|E|+|V|)\right)$.

\section{BEACON SET MINIMIZATION}

The second key problem—of minimizing the beacon set for a network-is formally stated below:

Beacon Minimization Problem (BMP). Let $D_{u}$ be the DMES associated with a node $u \in V$. Then the beacon-minimization problem is to find the smallest set of beacons, $B \subseteq V$, such that $\bigcup_{b \in B} D_{b}=E$.

Theorem 3. The Beacon Minimization Problem is NP-complete.

Proof: Let the graph under consideration be $G(V, E)$. Let $S$ be the set $\left\{D_{v}: v \in V\right\}$. Since every node can deterministically monitor at least its neighboring edges, $\bigcup_{v \in V} D_{v}=E$. Also, $D_{v} \subseteq E$. To find the smallest beacon set we need to find $B \subseteq S$ such that $\bigcup_{D_{v} \in B} D_{v}=E$ and $|B|$ is minimized. This is the the same as the classic Minimum Set Cover problem (MSCP) [13]. Thus, there is a one-to-one correspondence between BMP and MSCP, by using the concept of deterministically monitorable edge sets. The Minimum Set Cover Problem is known to be NP-Complete [13, 14]; this implies that BMP is NP-complete as well.

Fortunately, MSCP has a pruning-based approximate solutionbelow, we adapt the pruning algorithm and use heuristics from the literature to establish optimality bounds for it.

Algorithm 3. Find the beacon set for completely monitoring a graph $G(V, E)$.

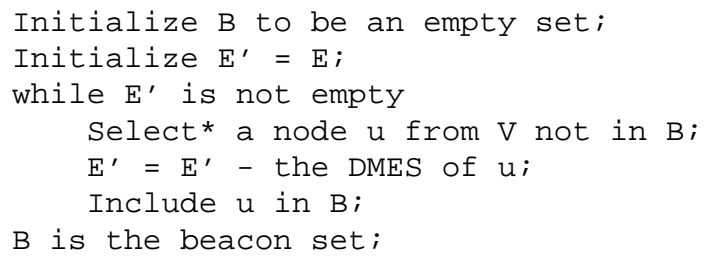

There exists a known heuristic for the MSCP pruning-based solution that ensures that the size of the solution is within a bound of the optimal [15]. The heuristic maps to the following nodeselection rule (* in above algorithm) for BMP. Select that node for a beacon whose DMES has the maximum overlap with the current pruned graph. Specifically, if $E^{\prime}$ is the current set of edges of the pruned graph then we choose the node $v$ such that $\left|D_{v} \cap E^{\prime}\right|$ is maximum. This heuristic results in provable [15] bounds of optimality of the beacon set as: $\frac{\mid B \text { (heuristic) }) \mid}{\mid B(\text { optimal }) \mid}=1+\ln |E|$.

\subsection{Further Optimizations}

We next establish additional monitoring-related properties of networks that let us further optimize the computation of the minimal beacon set. The concept of node arity in an undirected graph $G(V, E)$, defined in [7], is useful for this discussion. Below, we restate the definition from [7] in a slightly different manner.

Definition 2. (Node Arity) The arity of a node, $u$, with respect to another node, $v$, is defined as the number of distinct paths that exist between the two nodes such that each of these paths starts from a unique outgoing edge from $u$. The arity of a node $u$ is defined as the maximum of arities of $u$ with respect all nodes of the graph.

Using the terminology of [7], we call a node "high arity" if the node's arity is more than one. Note that since $G(V, E)$ is assumed to be connected, there is at least one path from every node to every other node (assuming $|V|>1$ ). Hence, the arity of a node 


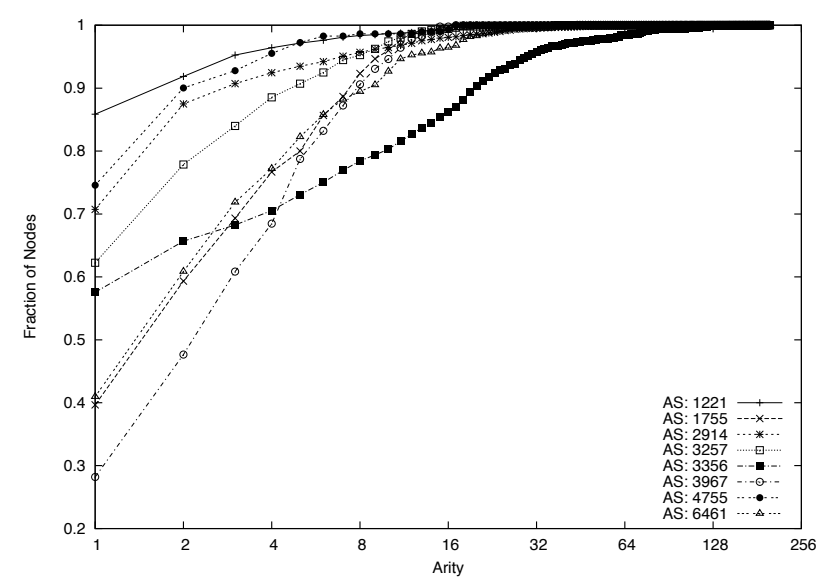

Figure 3: Cumulative distribution of node arities for eight Rocketfuel topologies.

is always greater than or equal to one. Also, since the maximum number of distinct paths (with a unique outgoing edge) from $u$ to $v$ can not be more than the degree of $u$, the arity of a node is bounded by its degree. In [9], we present an efficient algorithm for finding whether a node in $G(V, E)$ is high arity or not. Our algorithm is based on the insight that if a node $v$ has arity one, all forests generated in the depth-first search from $v$ will be connected to $v$ by a single edge.

The following theorems, proved in [9], establish useful properties of optimal beacon sets.

Theorem 4. A network with no high arity nodes can be monitored by a single beacon on any node in the network.

This theorem implies that a minimum beacon set can be computed trivially and optimally for any single-arity network (one that contains only single-arity nodes), without using the "pruning" algorithm presented earlier. In [9], we show that a single-arity network is a tree.

Theorem 5. An optimal beacon set, when beacons are simple or locally-flexible, is a subset of the set of high arity nodes.

Theorem 5 lets us reduce the set of potential beacons used in Algorithm 3 to the set of high arity nodes. This can lead to substantial computational savings. For instance, we show in Section 5 (and Figure 3), that the number and fraction of single arity nodes in current ISP topologies can be quite high.

In [7] the authors have shown that the set of high arity nodes in a graph is a beacon set-though potentially a non-optimal set-when beacons are locally-flexible. We have much strengthened this result by showing that the optimal beacon set is always a subset of the set of high-arity nodes (even with simple beacons). Not surprisingly, our pruning algorithm is able to find smaller beacon sets for all topologies. In order to numerically evaluate the efficacy of our formulations, we next present results of beacon set computations on a few real ISP topologies.

\section{EXPERIMENTAL RESULTS}

In this section, we compute and compare the beacons sets yielded for several current ISP topologies: (i) by the beacon placement solution with locally-flexible beacons suggested in [7]; (ii) by our beacon placement algorithms for simple beacons; and (iii) by our

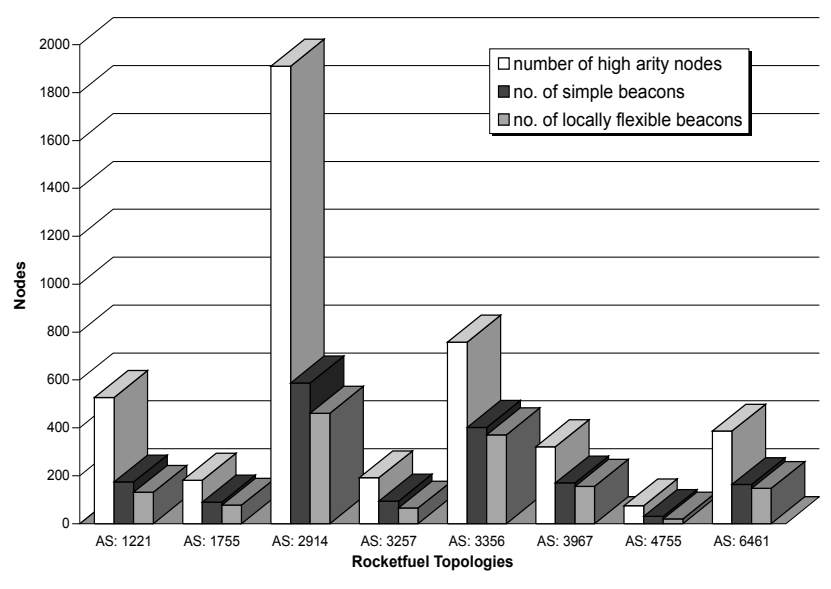

Figure 4: Histogram of beacon set sizes yielded by different algorithms for the Rocketfuel topologies.

algorithms for locally-flexible beacons. We refer to the resultant beacon sets as $B_{H A}, B_{S}$, and $B_{L F}$, respectively. We have implemented these algorithms in Java and have run these on eight major ISP topologies obtained from the Rocketfuel project at the University of Washington [8]. For each of the eight topologies, we analyze the distribution of node arities and calculate the sizes of beacon sets yielded by the three solutions.

Node Arities. The distribution of node arity for the eight topologies is plotted in Figure 3. We observe that:

1. The distribution of node arities are quite different for different ISPs, indicating that ISP topologies can be quite diverse in their topological structure. In particular, some ISP topologies have a long-tailed arity distribution, indicating that only a handful of nodes have significant redundancy in the manner in which they connect to the rest of the network. For most topologies, a majority of nodes have arities within 20 , although we some nodes can have arities higher than 150 .

2. The fraction of single arity nodes in the ISP topologies varies from less than $30 \%$ to more than $85 \%$. It is important to note that, for every other node in the network, a single arity node has only one local edge that can be used to reach it. Single arity nodes, therefore, are not robust to failures of local links. We find that for most topologies, more than half of the nodes have a single arity.

A large fraction of single-arity nodes also implies that the optimizations proposed in Section 4.1 to enable fast computation of beacon sets, can result in substantial savings.

It is important to observe that Rocketfuel ISP topologies are subject to inference errors. In particular, [16] demonstrates that the inclusion of links that do not exist and the omission of links that are actually present can inflate path diversity in these inferred topologies. This limits the accuracy of node arities computed above.

Beacon Set Sizes. It is important to mention that the Rocketfuel topologies for an ISP may not be connected (possibly due to lack of data about some links). Thus, some of the topologies we analyze have multiple (independent) connected components. More importantly, some of the components consist of only single-arity nodes (such components have a tree structure). For a fair comparison with 
the previous work in [7], which does not apply to single-arity networks, we ignore such components when computing beacon sets. ${ }^{6}$ For any ISP topology, we add up the sizes of beacon sets computed for each of the remaining components to get the total beacon set sizes- $\left|B_{H A}\right|,\left|B_{S}\right|$, and $\left|B_{L F}\right|$-for the three solutions being compared. Figure 4 plots the histograms of these beacon set sizes for the eight topologies. We observe that:

1. Beacon sets with locally-flexible beacons. Our beacon placement solution for locally-flexible beacons reduces the beacon set sizes yielded by [7] by $50-70 \%$. More importantly, we find that some major ISP topologies can be completely monitored, independent of routing state, using less than a hundred locally-flexible beacons. This is an encouraging observation as it suggests that a tomography-based monitoring infrastructure may not be infeasible even for major ISP topologies.

2. Beacon sets with simple beacons. Even with simple beacons, our beacon placement solution reduces the beacon set sizes of [7] by $40-70 \%$. This suggests that it may be feasible to design a simpler monitoring infrastructure that does not require that network nodes use different transmission rules for probe packets.

This conclusion is further supported by the comparison of beacon set sizes yielded by our solution for simple vs. locallyflexible beacons, which indicates that locally-flexible beacons may not yield significant gains for many major ISP topologies.

\section{FUTURE WORK}

There are several ways in which our work can be extended. We briefly discuss a few below.

In this paper, we consider two kinds of beacons: simple and locally-flexible. An important component of our future work is to generalize the notion of DMES for other kinds of beacons. For example, the beacons could form an overlay and use routing-tunnels to increase their DMES and reduce the beacon set size. We plan to explore the trade-off between beacon complexity and the beacon set size for monitoring realistic network topologies.

Our formulations assume that all high-level policies do not prohibit the use of a certain physical path between two network nodes. While this is a reasonable assumption for networks that are operated by a single autonomous entity, this may not be true when the network considered consists of multiple Autonomous Systems. In particular, multi-homed stub networks typically do not provide transit to traffic arriving from one of their ISPs and destined to a different ISP. We plan to extend our network model to incorporate such networks and compute beacon sets for large internetworks like the Internet.

An interesting extension of our framework is for monitoring infrastructure needed to monitor only a subset of all network links. For instance, an ISP may be interested in monitoring only its backbone or peering links. One approach for finding a beacon set for this scenario would be to create an abstraction in which some network nodes are collapsed to create a new network that contains only the relevant edges. The key challenges then would be in deciding where to install the beacons (as a single node in the collapsed graph may represent several nodes from the original network). We plan to explore this problem as part of future work.

${ }^{6}$ This eliminates only a small fraction of nodes from each of the ISP topologies.
Another interesting direction in which our work can be extended in by finding tree-like subgraphs in the network and use assigning one beacon to every such subgraph. The probes and/or routers would now need to be configured so that probe packets generating within a subgraph remain confined to the subgraph. This might be helpful in reducing the number of probes required, and the distance they travel, for monitoring all the edges of a large network.

\section{REFERENCES}

[1] CAIDA, "Skitter," http://www.caida.org/tools/ measurement/skitter.

[2] M. Coates, A. Hero, R. Nowak, and B. Yu, "Internet tomography," IEEE Signal Processing Magazine, May 2002.

[3] K. Claffy, T.E. Monk, and D. McRobb, "Internet tomography," Nature, 1999.

[4] Y. Bejerano and R. Rastogi, "Robust monitoring of link delays and faults in ip networks," in Proceedings of the 2003 ACM INFOCOM, 2003.

[5] N.G. Duffield and F. Lo Presti, "Multicast inference of packet delay variance at interior network links," in INFOCOM (3), 2000, pp. 1351-1360.

[6] N.G. Duffield, J. Horowitz, F. Lo Presti, and D. Towsley, "Network delay tomography from end-to-end unicast measurements," Lecture Notes in Computer Science, vol. 2170, pp. 576-??, 2001.

[7] J.D. Horton and A. Lopez-Ortiz, "On the number of distributed measurement points for network tomography," in Proceedings of the 2003 ACM SIGCOMM conference on Internet measurement, 2003, pp. 204-209.

[8] N. Spring, R. Mahajan, and D. Wetherall, "Measuring isp topologies with rocketfuel," in Proceedings of ACM/SIGCOMM '02, 2002.

[9] R. Kumar and J. Kaur, "Efficient beacon placement for network tomography," Technical Report, Department of Computer Science, University of North Carolina at Chapel Hill, August 2004.

[10] A. Shriram and J. Kaur, "Identifying bottleneck links using distributed end-to-end available bandwidth measurements," First ISMA Bandwidth Estimation Workshop, December 2003.

[11] Postel et. al., "Rfc 791: Internet protocol," Request for Comments, pp. 17-18, 1999.

[12] H.X. Nguyen and P. Thiran, "Active measurement for multiple link failures diagnosis in ip networks," in Proceedings of Passive and Active Measurement Workshop (PAM), April 2004.

[13] T.H. Cormen, C. Stein, R.L. Rivest, and C.E. Leiserson, Introduction to Algorithms, McGraw-Hill Higher Education, 2001.

[14] M.R. Garey and D.S. Johnson, Computers and Intractability: A Guide to the Theory of NP-Completeness, W. H. Freeman \& Co., 1979.

[15] T.H. Cormen, C.E. Leiserson, and R.L. Rivest, "Theorem 37.4, minimum set cover," Introduction to Algorithms, 1999.

[16] R. Teixeira, K. Marzullo, S. Savage, and G.M. Voelker, "In search of path diversity in isp networks," in Proceedings of the ACM SIGCOMM Internet Measurement Conference, October 2003. 